\title{
Design Consideration of Back-EMF Constant for 3-D.O.F. Spherical PM Motor
}

\author{
Sung-chul Go ${ }^{1}$, Dong-woo Kang ${ }^{1}$, Jong-bin $\mathrm{Im}^{1}$, Ju Lee ${ }^{1 *}$, Sung-hong Won ${ }^{2}$, and Seung-Bin Lim ${ }^{3}$ \\ ${ }^{1}$ Department of Electrical Engineering, Hanyang University, Seoul 133-791, Korea \\ ${ }^{2}$ Department of Electric System, Dongyang Technical College, Seoul 152-080, Korea \\ ${ }^{3}$ Korea Institute of Energy Technology Evaluation and Planning, Seoul 135-280, Korea
}

(Received 18 November 2009, Received in final form 5 March 2010, Accepted 5 March 2010)

\begin{abstract}
A 3-D.O.F. spherical PM motor has 3 degrees of freedom in its motion by tilting and rotating of a shaft, which can be applied in a range of fields. The back-EMF is proportional to the field flux and angular velocity. The back-EMF constant in conventional rotating machine has a uniform value. However, in a spherical PM motor, the back-EMF constant of the coils varies according to the tilting conditions regardless of whether the angular speed is constant. Consideration of the back-EMF constant is useful for designing 3-D.O.F. spherical PM motors. In this study, the back-EMF constant of the spherical PM motor was considered carefully.
\end{abstract}

Keywords : design, spherical motor, back-EMF, 3 degree of freedom

\section{Introduction}

The conventional classification of motors is divided into linear and rotary motors. Therefore, the system for multi-D.O.F. is a combination of motors. The PM motors need to be compact and have high power characteristics. This system employs a multi-frame structure with rotary motors for objected motion. However, a combination of many motors requires high power, volume and mass.

A spherical motor has been studied to overcome of the abovementioned shortcomings. The motor can be operated with 3-D.O.F. Therefore, the operation characteristics of the motor is different from conventional motors. However, classification by torque generation mechanism of a spherical motor is similar to the conventional motors [1-4].

The back-EMF is proportional to the linkage flux and angular velocity. The back-EMF constant is a useful indication of the operating characteristics in the motor design and control. It is possible that the back-EMF in a specified angular velocity and rated speed can determine the voltage, current and power. A back-EMF forward compensation algorithm can be used to control the motor, but the concept is not directly applicable to a spherical PM motor.

The magnitude of back-EMF has the same value for

*Corresponding author: Tel: +82-2-2220-0342

Fax: +82-2-2295-7111, e-mail: julee@hanyang.ac.kr each phase coil in conventional PM motors because of the static rotating plane. All back-EMF vectors have the same direction vector and the direction vector can be ignored. The shaft of the spherical PM motor can move through a spherical surface and rotate in a specified shaft position. Therefore, the magnet poles rotate in different rotating planes under tilt conditions. The coils have the same backEMF only when the shaft is positioned in the vertical direction. The magnitude of the back-EMF is not balanced in each phase coil if the shaft does not exist in that direction. This is due to the discordance coordinates between the coils and magnetic poles. Therefore, it is important to determine the back-EMF constant in a spherical PM motor using the back-EMF vector.

\section{Principle of Spherical PM Motor Classification}

\subsection{Basic concept of spherical motor}

It is difficult to explain the operating principle of a spherical PM motor because the shaft has movement in a spherical surface. Fig. 1(a) shows that the direction of torque in conventional PM motor is in the x-y plane. Fig. 1(b) shows that rotor of a spherical PM motor can move in 3-dimensions. The proper disposition of coils is essential for achieving correct positioning in the $x-y-z$ axes. At least 6 coils are necessary for the operation of a spherical PM motor. Hence, the spherical motor has rotating and 


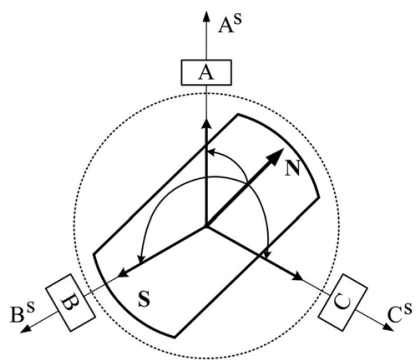

(a)

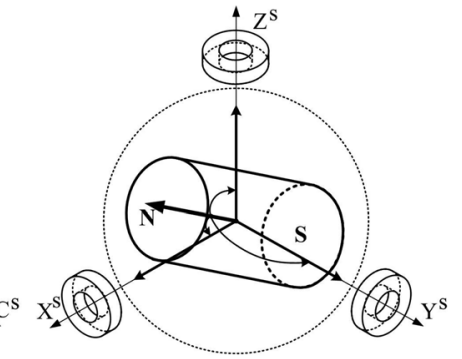

(b)
Fig. 1. Movement region of the rotor in conventional and spherical PM motors.

tilting characteristics.

\subsection{Structure Basic concept of spherical motor}

Two poles and 6 coils are needed to satisfy the spherical operation. Fig. 2(a) shows a stator with 12 coils in 2 layers. Fig. 2(b) shows a rotor composed 2 pole pairs. In addition, the shaft can only move in the open surface of the stator. Some frame structures are essential to sup-

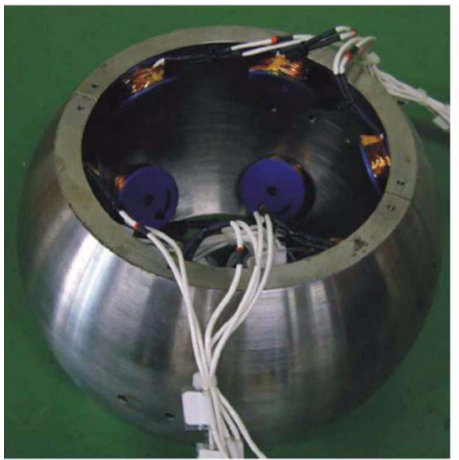

(a)

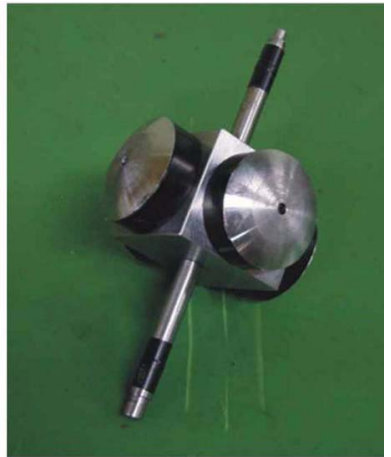

(b)
Fig. 2. Structure of a spherical PM motor.

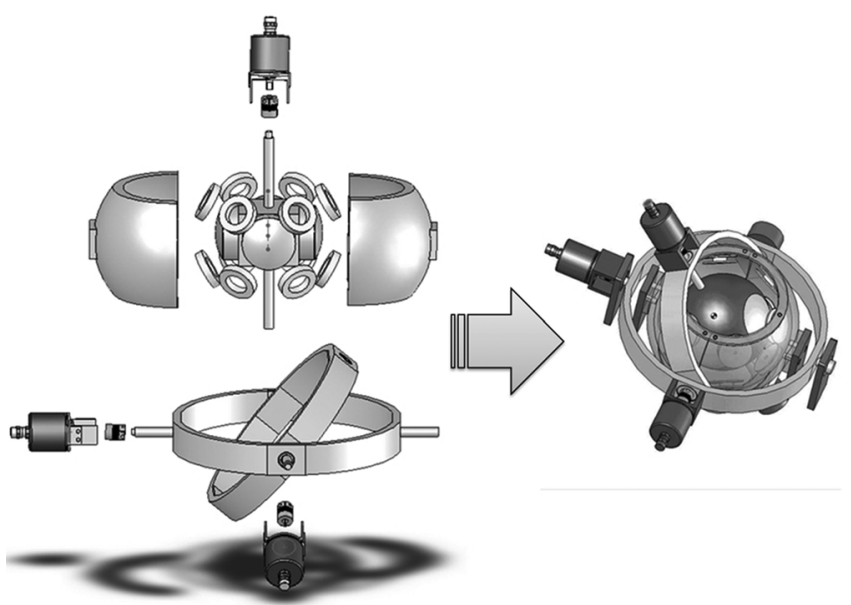

Fig. 3. Composition of a 4 pole spherical PM motor.

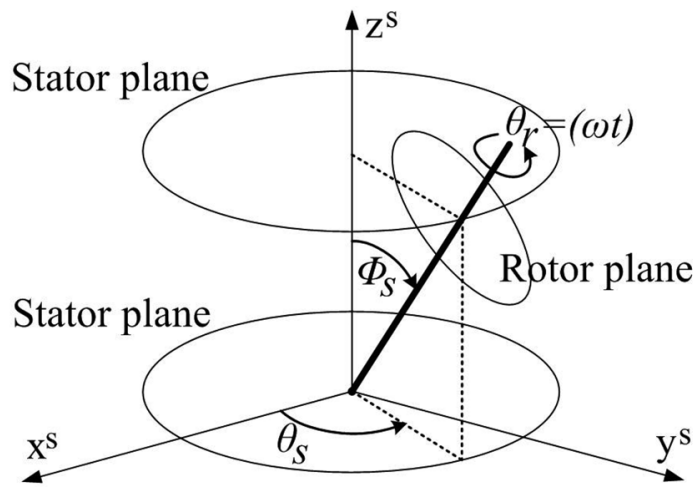

Fig. 4. Relationship between the shaft and stator plane of the spherical PM motor.

port rotor centering. Fig. 3 shows the precise composition of the spherical PM motor. No change in shaft position makes a stand-still of two frames. The shaft position is defined by the angles of $\Phi_{\mathrm{s}}, \theta_{\mathrm{s}}$, and $\theta_{\mathrm{r}}(\omega \mathrm{t})$ in Fig. 4. In this case, the shaft position is defined as angle $\Phi_{\mathrm{s}}$ from the z-axis first. The shaft position in the $x$-y plane is defined by $\theta_{\mathrm{s}}$. The rotation of the shaft is defined by $\theta_{\mathrm{r}}(\omega \mathrm{t})$. The parameter $\omega$ is the angular velocity. This angle $\theta_{\mathrm{r}}$ is related to the position of the magnet pole.

\subsection{Coordination transformation}

The position of the flux vector is the main element for obtaining the back-EMF. The coils are in the stator and the magnetic poles are in the rotor. A Z-Y-Z Euler transformation using Z-Y-Z Euler angles is a useful relative transformation [5]. The Z-Y-Z Euler transformation is shown in equation (1). Fig. 5 shows the $\alpha, \beta, \gamma$ rotation angles used in equation (1). The Z-Y-Z Euler transformation is a relative transformation. The vector position can be determined in different axes frames.

$$
[\Gamma]=\left[\begin{array}{c}
\cos (\alpha) \cos (\beta) \cos (\gamma)-\sin (\alpha) \sin (\gamma) \\
\sin (\alpha) \cos (\beta) \cos (\gamma)+\cos (\alpha) \sin (\gamma) \\
-\sin (\beta) \cos (\gamma)
\end{array}\right.
$$

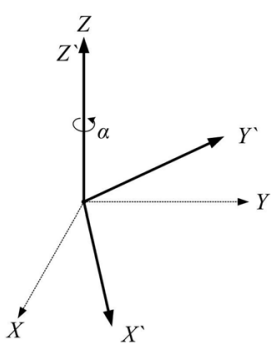

(a)

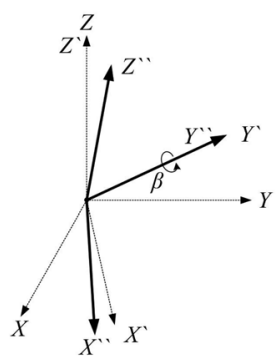

(b)

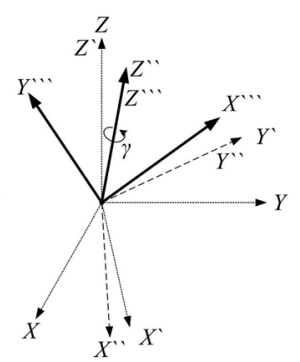

(c)
Fig. 5. Transformation by the Z-Y-Z transfer function. 


$$
\left.\begin{array}{cc}
-\cos (\alpha) \cos (\beta) \sin (\gamma)-\sin (\alpha) \sin (\gamma) & \cos (\alpha) \sin (\beta) \\
-\sin (\alpha) \cos (\beta) \sin (\gamma)+\cos (\alpha) \cos (\gamma) & \sin (\alpha) \sin (\beta) \\
\sin (\beta) \sin (\gamma) & \cos (\beta)
\end{array}\right]
$$

\section{Back-EMF Vector}

The magnitudes of the back-EMF in stator coils are the affected position of the shaft in a spherical PM motor. Therefore, the concept of the back-EMF constant is necessary in a spherical PM motor. The back-EMF vector calculation is necessary for the back-EMF constant. The back-EMF is related to the linkage flux and velocity. Accordingly, the position vector of the coils, the vector of the airgap magnetic density, and the line velocity in the coils are essential for calculating the back-EMF vector.

\subsection{Definition of position vector of coils}

The definition of the coil position is essential for obtaining the linkage flux and line velocity in coils. The spherical PM motor has a total of 12 coils. The spherical coordinate is useful for defining the position vector of the coils in the stator. Therefore, the vector is defined in equation (2).

$$
s=R_{s}[\sin (\phi) \cos (\theta) \sin (\phi) \sin (\theta) \cos (\phi)]^{T}
$$

where $R_{\mathrm{s}}$ denotes the semi diameter between the diagonal coils. It was assumed that $\mathrm{m}$ numbers of coils are dispatched with the $\delta_{\mathrm{c}}$ angle. The position vector of the $j$ coil is expressed in equation (3).

$$
\begin{aligned}
& s_{j}^{s}=R_{s}\left[\sin (\phi) \cos \left((j-1) \delta_{c}\right) \sin (\phi) \cos \left((j-1) \delta_{c}\right) \cos (\phi)\right]^{T} \\
& (j=1,2, \ldots, m)
\end{aligned}
$$

\subsection{Definition of magnetic density vector}

The magnetic density is related to the position of the magnetic pole and rotor [6]. Therefore, the position vector of the magnetic pole is important. It is assumed that $n$ numbers of magnetic poles are dispatched with a $\delta_{\mathrm{m}}$ angle around the shaft. The position vector of the $\mathrm{k}$ magnetic poles $r_{\mathrm{k}}{ }^{\mathrm{r}}$ is expressed as equation (4).

$$
\begin{aligned}
& r_{k}^{r}=R_{r}\left[\sin (\phi) \cos \left((k-1) \delta_{m}\right) \sin (\phi) \cos \left((k-1) \delta_{m}\right) \cos (\phi)\right]^{T} \\
& (k=1,2, \ldots, n)
\end{aligned}
$$

The position vector of the magnetic poles $r_{k}^{r}$ needs to transform to the stator plane because the induced EMF is calculated in the stator coils. Therefore, the position vector of the magnetic poles is transformed to orthogonal coordinates by the Z-Y-Z Euler angles (5).

$$
r_{k}^{s}=[\Gamma] R_{r}\left[\sin (\phi) \cos \left((k-1) \delta_{m}\right) \sin (\phi) \cos \left((k-1) \delta_{m}\right) \cos (\phi)\right]^{T}
$$

The vector of the magnetic flux density for back-EMF was calculated in the stator plane from $r_{k}^{r}$ and the magnitude of the magnetic flux density using equation (6).

$$
B_{k}^{s}=B_{\max } r_{k}^{s}=B_{\max }[\Gamma] r_{k}^{r}
$$

The linkage flux in the $j$ coil is obtained by the inner product of the magnetic flux density vector and position vector of the coils (7).

$$
\begin{aligned}
& \Psi_{j}^{s}=k_{\phi} \sum_{k=1}^{n} B_{k}^{s} \cdot r_{j}^{s} \\
& \left(j=1,2, \ldots, m, k_{\Phi} \text { is constant of linkage flux }\right)
\end{aligned}
$$

\subsection{Definition of back-EMF Vector}

The back-EMF vector was calculated by the outer product of the magnetic density vector and line velocity in coils. The line velocity was obtained first, as shown in Fig. 6. The unit direction vector of the rotor shaft is $u_{\mathrm{A}}$, the position vector of the coil is $u_{\mathrm{R}}$, and the vector of the line velocity is $u_{v}$. The relationship can be expressed as follows (8).

$$
u_{A}=u_{R} \times u_{v}
$$

Hence, $u_{v}$ is expressed as equation (9).

$$
u_{R} \times u_{A}=u_{R}\left(u_{v} \cdot u_{R}\right)-u_{v}\left(u_{R} \cdot u_{R}\right)=-u_{v}
$$

The unit vector of the line velocity of the $\mathrm{j}$ coil considers the angular velocity as follows $(10):(j=1,2, \ldots, m)$

$$
v_{j}^{s}=\omega\left(u_{A} \times s_{j}^{s}\right)
$$

The back-EMF vector in the coils was calculated as follows (11):

$$
e_{j}^{s}=\Psi_{j}^{s}\left(u_{R} \times v_{j}^{s}\right)
$$

Therefore, the back-EMF vector has a direction orthogonal to the direction of the position vector of the coils. The direction of rotation is constant. In addition, the upper coils and lower coils have the same direction of the

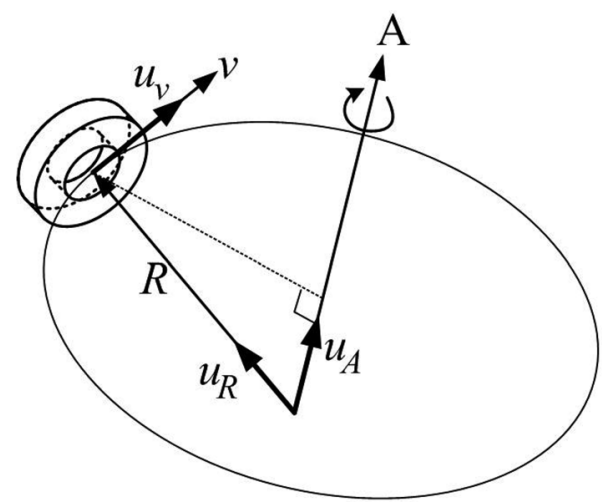

Fig. 6. Vector of the line velocity in a coil. 
$\mathrm{z}$-axis component. However, the upper coils have a central $\mathrm{x}$ and $\mathrm{y}$ axis direction. On the other hand, the lower coils are in the outer $\mathrm{x}$ and $\mathrm{y}$ axis direction of the backEMF vector because the back-EMF vectors are orthogonal to the position vector of the coils.

\subsection{Consideration of back-EMF constant}

In a conventional motor, the relationship between the coils and poles are not changed in the steady state [7]. The back-EMF vector in the stator coils is affected by the shaft position because of the magnetic pole. In a conventional rotating motor, each phase coils has the same direction of the back-EMF vector. Therefore, the direction has been ignored. However, only the magnitude of the backEMF cannot represent the spherical motor characteristic. The direction of the back-EMF vector is related to the position vector of the coil and rotating direction.

In different shaft tilt conditions, the magnitude of the back-EMF in each coil has a different value. Therefore, the same speed condition does not mean the same backEMF measurement in each coil. Therefore, the back-EMF vector must be considered carefully. The three direction element of the back-EMF vector exists in the stator reference frame. In addition, the component in the shaft tilt condition was varied. The back-EMF is produced by coils and a magnet. The magnet direction affects the backEMF. In addition, the position of the magnet is fixed in the rotor. Therefore, the back-EMF was considered in the rotor reference frame. In this case, the z-axis of the rotor is the direction of the shaft. In addition, the back-EMF components of the $\mathrm{x}$ and $\mathrm{y}$ axes are almost zero due to the back-EMF vector direction. Consequently, the component of the $\mathrm{z}$-axis is the effective back-EMF vector in the rotor plane. The vector is transformed to the rotor reference frame. The Z-Y-Z Euler angles are as follows (12):

$$
\begin{aligned}
& \alpha=\theta_{s} \\
& \beta=\phi_{s} \\
& \gamma=\theta_{r}-\theta_{s} \cos \phi_{s}
\end{aligned}
$$

The element of the $\mathrm{z}$-axis in the rotor reference frame is expressed as (13).

$$
e_{Z}^{s}=\left(\sum_{j=1}^{m}\left(u_{A} \cdot[\Gamma] e_{j}^{s}\right)\right) u_{A}
$$

The back-EMF constant was calculated by the backEMF in the rotor shaft axis over the angular velocity (14).

$$
k_{e}^{r}=\frac{e_{z}^{r} \cdot u_{A}}{\omega}
$$

In this case, the back-EMF constant is unaffected by the change in shaft position. Therefore, this concept is considered appropriate in a spherical PM motor.

In a spherical PM motor, the back-EMF constant means the maximum back-EMF in the range of all operations. In the vertical shaft position, rotation produces a lower backEMF than that back-EMF calculated from the proposed back-EMF constant. However, some coils have a maximum back-EMF in the hard tilting operation condition.

\section{Measurement of Back-EMF Constant}

The back-EMF constant was verified by measuring the back-EMF. The back-EMF measurement system is composed of a spherical PM motor, load motor, and PXI instrument, as shown in Fig. 7. The PXI instrument is operated using Labview language and a FPGA module for data acquisition. Table 1 shows the specification of the test bed. The 4 poles spherical PM motor has basically 12 coils in two layers. Therefore, each coil has a different effect of magnet of rotor rotation. Therefore, it is impossible to consider all cases. Three cases were selected to consider the back-EMF in different shaft positions. Fig. 8 shows the cases. The 12 coils were named with $\mathrm{AU}, \mathrm{BU}$, $\mathrm{CU}, \mathrm{DU}, \mathrm{EU}, \mathrm{FU}, \mathrm{AD}, \mathrm{BD}, \mathrm{CD}, \mathrm{DD}, \mathrm{ED}$, and $\mathrm{FD}$ in $60\left(^{\circ}\right)$

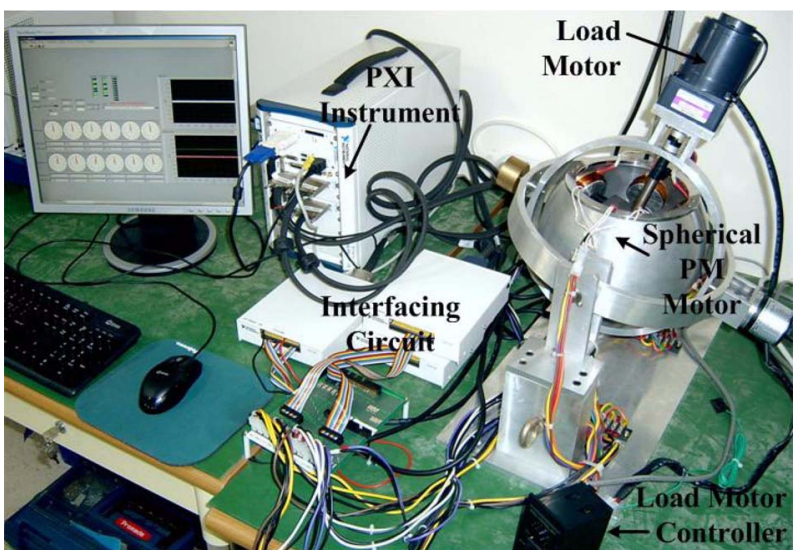

Fig. 7. Back-EMF measurement system of a spherical PM motor.

Table 1. Specification of the test bed.

\begin{tabular}{ccc}
\hline \hline Test system & $\begin{array}{c}\text { Load motor } \\
\text { Torque transducer } \\
\text { Back-EMF } \\
\text { measurement }\end{array}$ & $\begin{array}{c}\text { BLDC Motor (700W)+Controller } \\
\text { None }\end{array}$ \\
\hline Test target & Spherical PM Motor & 4 poles, 12 coils \\
\hline \multirow{3}{*}{ Test points } & Angular speed & $132[\mathrm{rpm}]$ \\
& Case I & no tilt \\
& Case II & Tilt to negative x-axis \\
& Case III & Tilt to negative y-axis \\
\hline
\end{tabular}


Table 2. Magnitude of Back-EMF in upper coils (132[rpm]).

\begin{tabular}{ccccccc}
\hline \hline Case & AU Coil [V] & BU Coil [V] & CU Coil [V] & DU Coil [V] & EU Coil [V] & FU Coil [V] \\
\hline Case I & 0.4585 & 0.4323 & 0.4768 & 0.4503 & 0.3945 & 0.4441 \\
Case II & 0.6041 & 0.5256 & 0.3650 & 0.2005 & 0.3349 & 0.5119 \\
Case III & 0.4951 & 0.5584 & 0.5524 & 0.4829 & 0.2107 & 0.2111 \\
\hline
\end{tabular}

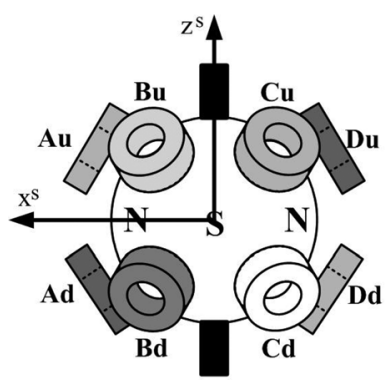

Case I

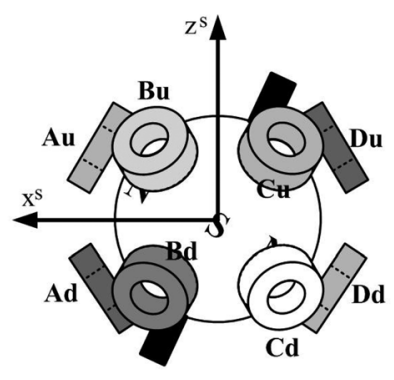

Case II

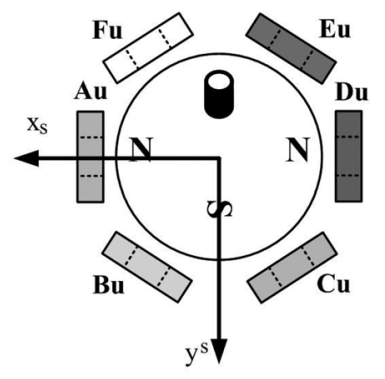

Case III

Fig. 8. Determination of 3 cases for a back-EMF consideration.

intervals and 2 layers. The notation, $\mathrm{AU}$, means the A phase and upper coils. The notation, FD, means the $\mathrm{F}$ phase and lower coils. The cases were determined to consider the various back-EMF magnitudes of 12 coils. Case I is the no tilt condition of the shaft, $\Phi_{\mathrm{s}}=0\left({ }^{\circ}\right) \theta_{\mathrm{s}}=0\left({ }^{\circ}\right)$. Therefore, the magnet pole affects the same magnitude of the back-EMF to each coil. The condition of case II was tilted by the $y$-axis negative rotation in the rotor reference frame $\Phi_{\mathrm{s}}=18\left(^{\circ}\right) \theta_{\mathrm{s}}=180\left(^{\circ}\right)$. Hence, the magnet pole is closest to the AU and DD coils, and magnitude of the back-EMF is highest in those coils. Case III is the tilt to the negative y-axis, $\Phi_{\mathrm{s}}=18\left(^{\circ}\right) \theta_{\mathrm{s}}=270\left(^{\circ}\right)$. Therefore, the magnet pole is closest to the middle point of the $\mathrm{BU}$ and $\mathrm{CU}$ coils and the back-EMF magnitude reaches a peak in the BU, CU, ED and FD coils.

The system receives an encoder signal and back-EMF signal from a motor with interfacing. The configuration for the measurement was divided into 3 cases. Fig. 9 shows the back-EMF waveform of 3 cases. Fig. 9(a) and (b) presents the results of case I. Fig. 9(c) and (d) is shows case II and Fig. 9(e) and (f) shows case III. Table 2 shows the effective value of the back-EMF in the upper coils. The back-EMF was highest in the AU coil in the case II measurement.

The back-EMF constant was obtained by the back-EMF over speed. In addition, the constant did not change under the linear magnetic saturation region in a conventional motor. However, the back-EMF magnitudes of the coils are different in cases II and III. Therefore, the back-EMF was calculated in the in the rotor reference frame. In the case I, the $\alpha$ and $\beta$ Euler angles are both $0^{\circ}$. In the case II, the $\alpha$ the $\beta$ Euler angle is $18\left(^{\circ}\right)$ and $180\left({ }^{\circ}\right)$, respectively. In the case III, the $\alpha$ and $\beta$ Euler angle is $18\left(^{\circ}\right)$ and $270\left(^{\circ}\right)$, respectively. The $\gamma$ Euler angle was determined by the position angle between the magnet and each coil. The back-EMF vector was calculated using equation (1) with the angles. Table 3 lists the back-EMF constant in the rotor $x-y-z$ directions and the units are voltage per $\mathrm{rad} / \mathrm{s}$. The Euler angles were determined by the shaft position. The back-EMF constant of the z-axis was almost constant in the three cases. The difference in the z-axis back-EMF constant in Fig. 9 was considered to be measurement error. The back-EMF constant in the rotor reference frame was transferred from the value in the stator reference frame by Euler's transformation.

\section{Conclusion}

The magnitude of the back-EMF in a spherical PM motor varies according to the shaft position. It is not possible have a constant back-EMF in the rated speed of a spherical PM motor due to shaft movement. The advance of a spherical PM motor varies according to the operating condition in same speed rotation. However, the maximum back-EMF is important under the condition of rated rotation. A proper back-EMF constant consideration is a fixed back-EMF constant. In addition, the back-EMF is determined by magnet poles rotating frame. In a conventional rotating motor, the direction of the back-EMF was ignored because of the same direction. However, in a 


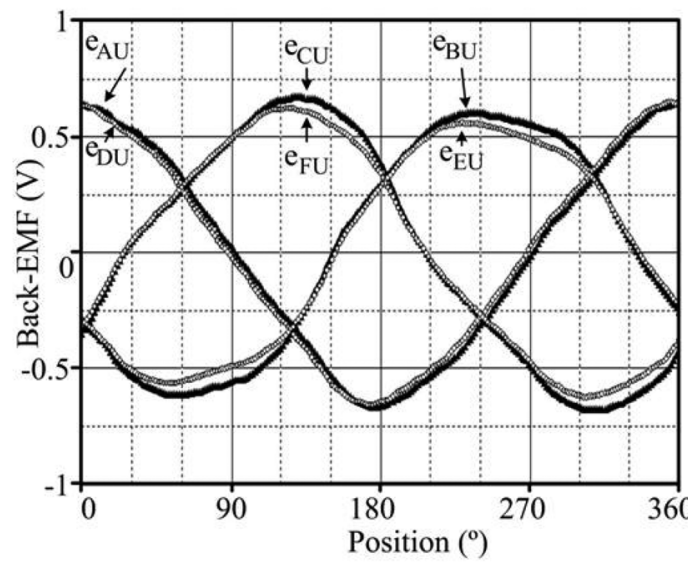

(a)

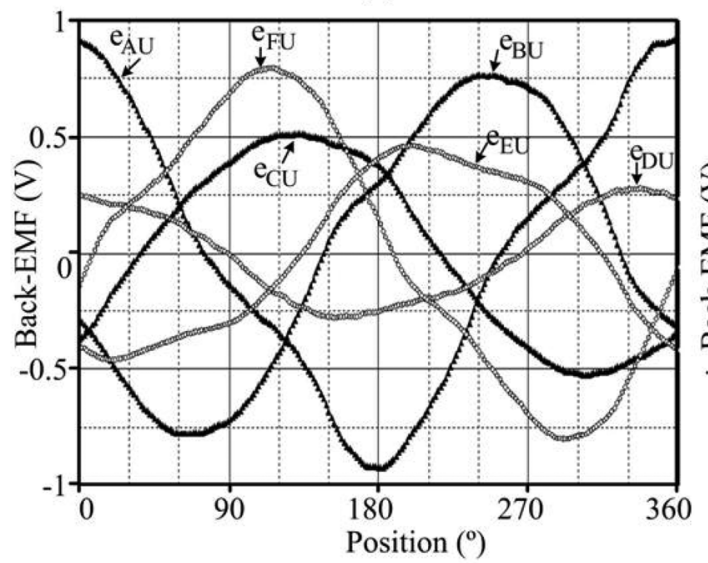

(c)

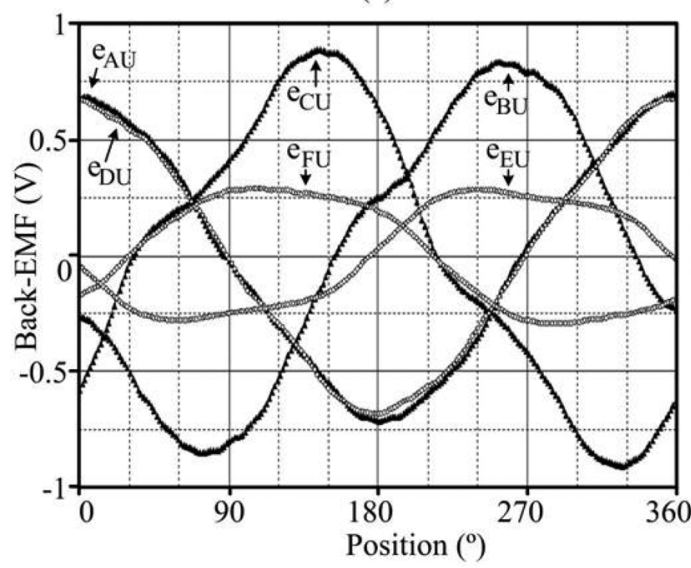

(e)

Fig. 9. Back-EMF waveform in three cases.

Table 3. Back-EMF constant in rotor reference frame.

\begin{tabular}{cccccc}
\hline \hline \multirow{2}{*}{ Case } & \multirow{2}{*}{$\begin{array}{c}\text { Euler angles (Degree) } \\
\text { Back-EMF constant (V/(rad/s)) } \\
\text { In rotor reference frame }\end{array}$} \\
\cline { 2 - 6 } & $\alpha$ & $\beta$ & X-axis & Y-axis & Z-axis \\
\hline Case I & 0 & 0 & 0.0000 & 0.0000 & 0.0154 \\
Case II & 180 & 18 & 0.0042 & 0.0008 & 0.0147 \\
Case III & 270 & 18 & 0.0000 & 0.0037 & 0.0148 \\
\hline
\end{tabular}

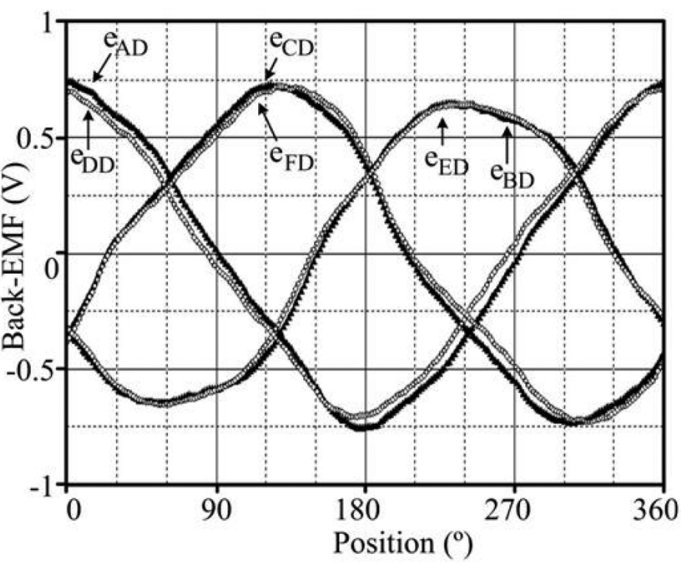

(b)

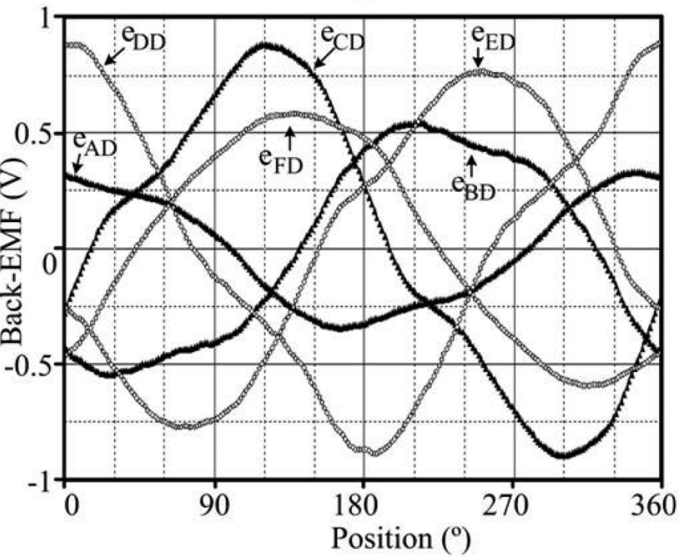

(d)

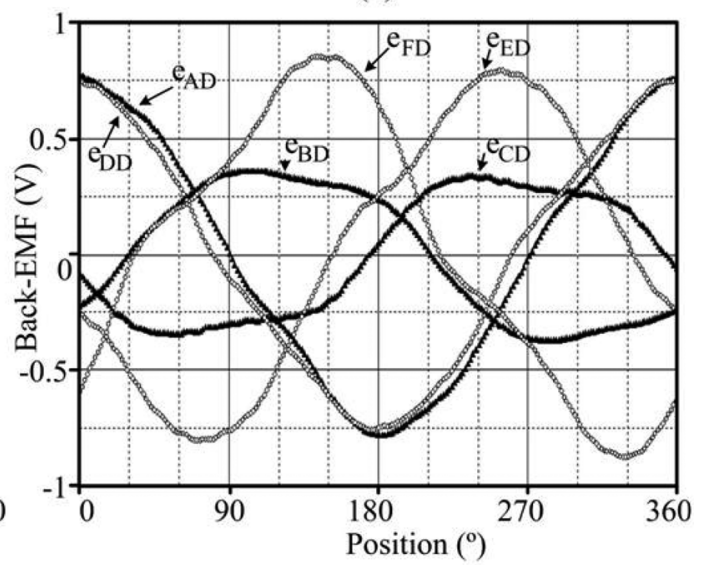

(f)

spherical PM motor, the direction of the back-EMF in each coil must be considered. The shaft direction varies according to the operation. The back-EMF is affected by the shaft position. In this case, the spherical PM motor designer must consider the limitation of the maximum operation. Therefore, the back-EMF vector can be used to indicate the motor design characteristics. The back-EMF 
constant is affected by the shaft position. This consideration was verified by measuring the back-EMF in the three cases of the shaft position. Overall the back-EMF constant in a spherical PM motor is useful for designing and controlling the motor.

\section{Acknowledgment}

This study was supported by the National Research Laboratory (NRL) through a grant provided by the Ministry of Education, Science \& Technology (MEST, contract number R0A-2008-000-20048-0), and partially by the Manpower Development Program for Energy \& Resources supported by the Ministry of Knowledge and Economy (MKE), Republic of Korea.

\section{References}

[1] F. Williams, E. Laithwaite, and J. F. Eastham, Proc. Inst. Elec. Eng. 106A, 471 (1959).

[2] T. Yano, T. Suzuki, M. Sonoda, and M. Kaneko, Proc. 1999 IEEE/RSJ Int. Conf. Intelligent Robots and Systems, 1393 (1999).

[3] B. B. Bederson, R. S. Wallace, and E. L. Schwartz, Proc. 1993 IEE Int. Conf. Robotics and Automation, Vol. 2, 630 (1993).

[4] S. Toyama, S. Hatae, and M. Nonaka, Int. Conf. Advanced Robotics 1, 55 (1991).

[5] John J. Craig, Introduction to Robotics, Pearson Prentice Hall, Englewood Cliffs (2005).

[6] Changliang Xia, Hongfeng Li, and Tingna Shi, IEEE Trans. Magn. 44, 2016 (2008).

[7] Jhih-Da Hsu, and Ying-Yu Tzou, IEEE Power Electronics Specialists Conf., 939 (2007). 